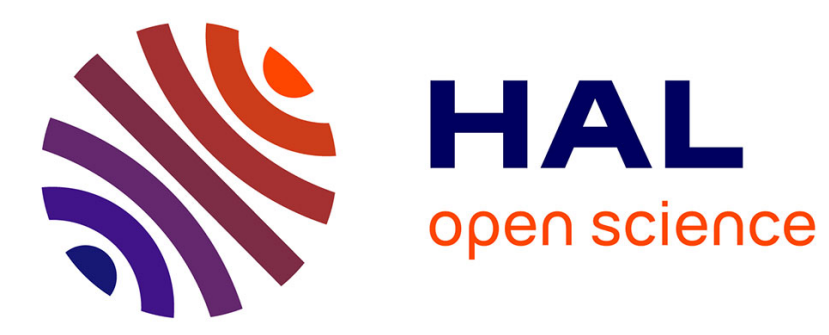

\title{
La Revue STAPS et l'interdisciplinarité : une étude socio-informatique
}

Cécile Collinet, Terral Philippe, Patrick Trabal

\section{To cite this version:}

Cécile Collinet, Terral Philippe, Patrick Trabal. La Revue STAPS et l'interdisciplinarité : une étude socio-informatique. STAPS : Revue internationale des sciences du sport et de l'éducation physique, 2012, 96-97 (2), pp.29. 10.3917/sta.096.0029 . hal-03017034

\section{HAL Id: hal-03017034 \\ https://hal.parisnanterre.fr/hal-03017034}

Submitted on 20 Nov 2020

HAL is a multi-disciplinary open access archive for the deposit and dissemination of scientific research documents, whether they are published or not. The documents may come from teaching and research institutions in France or abroad, or from public or private research centers.
L'archive ouverte pluridisciplinaire HAL, est destinée au dépôt et à la diffusion de documents scientifiques de niveau recherche, publiés ou non, émanant des établissements d'enseignement et de recherche français ou étrangers, des laboratoires publics ou privés. 


\title{
La Revue STAPS et l'interdisciplinarité : une étude socio-informatique « STAPS » journal and interdisciplinarity: a socio-computer study
}

\author{
Collinet Cécile • Terral Philippe • Trabal Patrick
}

RéSumÉ : À partir d'une analyse de 602 articles de la Revue STAPS publiés entre 1980 et 2010, s'appuyant sur le logiciel Prospero, la recherche se propose de repérer la pluralité des formes que peut prendre l'interdisciplinarité au sein des textes scientifiques. La préoccupation d'interdisciplinarité apparaît constante et sa difficile reconnaissance est soulignée. On rencontre aussi bien des allusions superficielles à la notion que des revendications. L'interdisciplinarité est alors présentée comme pertinente pour éclairer certains objets complexes, rapprocher les recherches de la demande sociale, favoriser la productivité ou la créativité scientifique. Le mot d'ordre d'interdisciplinarité peut également être associé à des logiques de défense territoriale de courants de pensée menacés. Plus rarement, l'interdisciplinarité est prise comme objet d'étude voire critiquée. Lors de l'analyse des formes de cohabitation de lexiques disciplinaires dans un même article, nous repérons ensuite les mélanges plus ou moins fréquents. On note que les questions d'enseignement génèrent la convocation d'au moins deux lexiques disciplinaires. Enfin, qu'il s'agisse d'opérations de contextualisation de l'objet de recherche via une autre discipline ou de discussions critiques entre deux domaines de connaissance, nous soulignons que l'activité interdisciplinaire engage dans les textes eux-mêmes un travail réflexif sur les façons de faire de la science.

MoTS CLÉS : Interdisciplinarité, STAPS, articles, Prospéro.

ABSTRACT: Since an analysis with Prospero software of 602 articles published in STAPS journal between 1980 and 2010, our research identifies the diversity of interdisciplinary forms in scientific texts. The preoccupation of interdisciplinarity is constant even if it acknowledgement seems difficult. Our analysis also detects superficial allusions than demands. Interdisciplinarity is then showed as an efficient

DOI: $10.3917 /$ sta.096-097.0029

Cécile COLLINET

Université Paris-Est Marne-la-Vallée Laboratoire ACP 5 bd Descartes 77454 Champs-sur-Marne cecile.collinet@univ-mlv.fr

Téléphone professionnel : +33 (0)160957578 (71)

Professeur

Philippe TERRAL

Université Paul Sabatier Laboratoire PRISSMH 118 route de Narbonne 31062 Toulouse cedex 9

philippe.terral@univ-tlse3.fr

Téléphone personnel : +33 (0)6 67674580

Téléphone professionnel : +33 (0)561557342

Maître de conférences

Patrick TRABAL

Université Paris-Ouest Nanterre La Défense Laboratoire CERSM, 200 avenue de la République, 92000 Nanterre patrick.trabal@u-paris10.fr 
way to understand complex subjects, to connect researches with social demand, to generate scientific productivity or creativity. The demand of interdisciplinarity is also associated to a territorial defense from some scientific tendencies less recognized. Rarely, interdisciplinarity is too considered as an object of analysis or could also be criticized. When we consider forms of cohabitation of diverse disciplinary vocabulary in a same article, we identify more or less frequent mixes. We notice that the questions of teaching generate the association of a minimum of two disciplinary vocabularies. Finally, considering operations of contextualization of a research object from another disciplinary point of view or a critical discussion within two disciplinary tendencies, the interdisciplinarity work in scientific articles looks like a reflexive activity of the way to produce science

KEYWORDS: Interdisciplinarity, STAPS, articles, Prospero.

\section{Zusammenfassung: Die Zeitschrift STAPS und die Interdisziplinarität. Eine wissenssoziologische Studie}

Anhand einer computergestützten Analyse mit dem Programm Prospero von 602 Artikeln der Zeitschrift STAPS, die zwischen 1980 und 2010 veröffentlicht wurden, will die vorliegende Untersuchung die Pluralität der Formen aufzeigen, welche die Interdisziplinarität in wissenschaftlichen Texten annehmen kann. Die Sorge um die Interdisziplinarität ist beständig vorhanden und die Schwierigkeit ihrer Anerkennung wird betont. Der Begriff ist Gegenstand oberflächlicher Andeutungen, aber auch von Forderungen. Die Interdisziplinarität wird dann als nützlich dargestellt, um bestimmte komplexe Gegenstände zu beleuchten, die Forschung der sozialen Nachfrage anzupassen, die Produktivität oder die wissenschaftliche Kreativität zu fördern. Die Forderung nach Interdisziplinarität kann auch mit einer Logik in Verbindung gebracht werden, bei der es darum geht bedrohte Forschungsgebiete zu verteidigen. Seltener wird die Interdisziplinarität selbst zum Forschungsobjekt oder gar zum Gegenstrand von Kritik. Bei der Analyse der Kohabitation von disziplinärem Vokabular in ein und demselben Artikel bestimmen wir die Häufigkeit der Mischungen. Es ist dabei anzumerken, dass die Fragestellungen von mindestens zwei unterschiedlichen disziplinären Vokabularien ausgehen. Schließlich möchten wir unterstreichen, dass, egal ob es sich um Operationen der Kontextualisierung des Forschungsobjektes durch eine andere Disziplin handelt oder um kritische Diskussionen zwischen zwei Wissensbereichen, die interdisziplinäre Arbeit in den Texten selbst eine reflexive Arbeit über die Art Forschung zu betreiben hervorbringt.

SCHLAGWÖRTER: Interdisziplinarität, STAPS, Artikel, Prospero.

\section{RIAsSunto: La rivista STAPS e l'interdisciplinarietà: uno studio socio-informatico}

A partire dall'analisi dei 602 articoli della rivista STAPS pubblicata tra il 1980 e il 2010 basandosi sul programma Prospero, la ricerca si propone di reperire la pluralità delle forme che può prendere l'interdisciplinarietà in seno ai testi scientifici. La preoccupazione di interdisciplinarietà appare costante ed è sottolineato il suo difficile riconoscimento. Si incontrano anche delle allusioni superficiali alla nozione delle rivendicazioni. L'interdisciplinarietà è allora presentata per chiarire certi oggetti complessi, avvicinare le ricerche alla domanda sociale, favorire la produttività o la creatività scientifica. La parola d'ordine di interdisciplinarietà può ugualmente essere associata a logiche di difesa territoriale di correnti di pensiero minacciate. Più raramente, l'interdisciplinarietà è presa come oggetto di studio perfino criticato. Durante l'analisi delle forme di coabitazione di lessici disciplinari in uno stesso articolo, in seguito reperiamo le mescolanze più o meno frequenti. Si nota che le questioni d'insegnamento generano la convocazione di almeno due lessici disciplinari. Infine, che si tratti di operazioni di contestualizzazione dell'oggetto di ricerca attraverso un'altra disciplina o di 
discussioni critiche tra due ambiti di conoscenza, noi sottolineiamo che l'attività multidisciplinare richiede nei testi stessi un lavoro riflessivo sui modi di fare della scienza.

PAROLE CHIAVE: Articoli, interdisciplinarietà, scienza, STAPS.

\section{ResUMEN : La revistas STAPS y la interdisciplinaridad : un estudio socio informático}

A través del programa informático prospero se analizaron 602 artículos de la revista STAPS publicados entre los años 1980 y 2010, la investigación propuesta es revisar en los textos científicos la interdisciplinaridad. La preocupación por la interdisciplinaridad es constante y de difícil reconocimiento. Se pueden observar algunas alusiones superficiales en relación a la noción de reivindicación La interdisciplinaridad se presenta como pertinente en clarificar algunos objetos complejos, aproximar la investigación a la demanda social puede favorecer la productividad o bien la creatividad científica. La palabra orden interdisciplinaria se puede asociar a lógicas de defensa territorial en algunas corrientes de pensamiento amenazadas. Raramente, la interdisciplinaridad es considerada como un objeto de estudio con mirada crítica. En un análisis en formas de cohabitación de lexicos disciplinarios en un mismo artículo, se observan las mezclas más o menos frecuentes. Se puede notar que las interrogantes de enseñanza pueden generar una convocación de más lexicos disciplinarios. Cuando se trata de operaciones de contextualización del objeto de la investigación a través de una disciplina o mediante una discusión crítca entre dos dominios del conocimiento, señalamos que la actividad interdisciplinaria considerada en los textos es un trabajo reflexivo como una forma de realizar ciencia.

PaLABRAS Claves : Interdisciplinaridad, STAPS, artículos, Prospero.

\section{INTRODUCTION}

L'interdisciplinarité n'est pas une thématique nouvelle dans les réflexions sur la science. Elle émerge dans les années 1970 au moment des grandes réformes de l'enseignement supérieur qui portent en elles une critique de l'organisation cloisonnée des disciplines scientifiques et qui s'appuient sur l'idée que l'organisation des savoirs en disciplines se heurte à une réalité complexe qui, elle, n'est pas disciplinaire. L'organisation d'un colloque à Nice par l'OCDE en 1972 sur ce thème illustre les questionnements qui émergent et les tentatives de définir ce que pourrait être un travail scientifique interdisciplinaire. Les auteurs (i.e. Boisot, Heckhausen, 1972 ; Jantsch, 1972 ou plus récemment Sinaceur, 1992; Vinck, 2000 ; Frodeman et al., 2010; Prud'homme et al., 2012) rivalisent de propositions en matière de classifications censées caractériser les différentes formes du travail interdisciplinaire et établissent une sorte de continuum entre un travail qui consisterait à juxtaposer des disciplines et un autre qui les mêlerait plus profondément. La classification tripartite de Piaget (1972) entre une pluri-, une inter- ou une transdisciplinarité illustre cette volonté de graduation du travail interdisciplinaire. Pour servir l'analyse, il a été choisi de prendre le terme « interdisciplinarité » dans un sens générique recouvrant les différents modes de relations entre disciplines afin de ne pas qualifier de manière aprioriste leurs manières de collaborer.

L'interdisciplinarité semble aujourd'hui encore un thème central dans les réflexions sur l'organisation scientifique (voir, par exemple, Maasen \& Lieven, 2006), particulièrement activé depuis les différentes réformes de l'enseignement supérieur et de la recherche dans la première décennie des années 2000. Un rapide coup d'œil sur la presse permet de confirmer que l'interdisciplinarité est une solution largement convoquée lorsqu'il s'agit de proposer une réforme de l'université ou de nouvelles formes de travail scientifique. Ainsi, par 
exemple, en avril 2011, le compte rendu de la réforme de l'université de Rennes pointet-il que «l'heure est donc au rapprochement et à l'interdisciplinarité " ( Les lauriers de l'université ", Le Point, 7 avril 2011). En 2010, le journaliste J.-C. Lewandowski écrit que l'on assiste à « une sorte de révolution silencieuse qui se déroule sur les campus. Son nom? Interdisciplinarité. En clair, il s'agit de multiplier échanges et collaborations entre différents domaines : le marketing et le design, l'informatique et la sociologie, la médecine et les sciences de l'ingénieur...» (« Le temps des cursus pluridisciplinaires », Les Échos, 30 novembre 2010). Ce même auteur signalait que parmi les six compétences essentielles du chercheur idéal figurait l'interdisciplinarité ("Le profil idéal du chercheur ", Les Échos, 9 novembre 2010). Ainsi « le milieu de la recherche, longtemps connu pour ses cloisonnements, a amorcé sa mue. L'heure est à l'“interdisciplinarité", aux frottements entre disciplines jugées propices à l'innovation " (Jacques Trentesaux, "Saclay Le futur sur un plateau ", L'Express, 17 novembre 2010). L'organisation en PRES (Pôle de Recherche et d'Enseignement Supérieur) des universités est censée la promouvoir: "Pour cela, il faudra que les P.r.e.s. soient capables de transformer en collaborations scientifiques plus efficaces les rapprochements administratifs. Ces coopérations peuvent être favorisées par des écoles doctorales plus larges, une politique scientifique plus forte des universités et surtout une interdisciplinarité dépassant les clivages antérieurs entre établissements, comme entre sciences humaines et sciences de la nature. » (Sylvestre Huet, "Une recherche plus efficace?", Libération, 17 mars 2010). Ce rapide tour d'horizon n'est pas exhaustif mais rend bien compte de l'actualité du thème ; actualité perceptible aussi dans les réflexions sur le travail scientifique, notamment de langue anglaise (Klein, 1990 ; Stehr \& Weingart, 2000 ...).

Si l'interdisciplinarité est une thématique importante, dans les discours sur le travail scientifique perceptible entre autres dans la presse qui se fait souvent le relais de la rhétorique gouvernementale, mais aussi dans les projets scientifiques, les programmes de recherche des laboratoires... il n'en reste pas moins que les disciplines restent une référence incontournable de l'organisation de l'enseignement mais aussi de la recherche et du travail scientifique proprement dit (Collinet, 2003). Sûrement faut-il y voir le poids historique de la constitution et du développement des disciplines. Le mode de fonctionnement scientifique et les dispositifs qui lui sont liés se définissent souvent par rapport à une discipline : la participation à des comités de sélection, les profils de postes, la désignation d'experts pour l'évaluation des laboratoires ou des revues, l'écriture et la publication scientifiques... mobilisent la plupart du temps des disciplines identifiées et posent la question de l'identification de celles-ci et de la délimitation de territoires disciplinaires.

Cette posture complexe entre disciplinarité et interdisciplinarité se pose de manière particulièrement intéressante dans le domaine des STAPS, section mêlant historiquement plusieurs disciplines. Cette thématique a fait l'objet de nombreuses réflexions dans le domaine, anciennes (par exemple, Prévost, 1988) ou plus récentes (Collinet, 2003 ; Quidu, 2012).

Que la pluralité soit envisagée de manière enrichissante ou pénalisante, elle est assez souvent un sujet de discussion important structurant l'activité épistémologique du domaine. Ainsi, dès les années 1980, période de structuration universitaire fondamentale pour les STAPS et la recherche en son sein, la question de l'interdisciplinarité est posée :

«Les APS constituent par leur diversité un carrefour pluridisciplinaire ou dans le meilleur des cas, interdisciplinaire, c'est-à-dire tenant compte des influences réciproques des diverses disciplines. Enseigner les STAPS, quelle que soit son origine, c'est donc obligatoirement avoir la préoccupation de ne pas réduire la relation éducative ou le fait éducatif pris dans un sens très large, à une seule composante : la sienne» (Thibault, 1981). 
La pluridisciplinarité scientifique est souvent revendiquée comme étant une caractéristique importante du domaine. Elle impacte fortement les parcours de formation des enseignants-chercheurs formés en interne (Collinet, 2003 ; Collinet \& Payré, 2004). Ces derniers ont en effet été socialisés par diverses disciplines et n'ont choisi de spécialité disciplinaire de recherche que relativement tard lors de leur orientation en master pour certains ou encore plus tardivement au moment de leur doctorat pour d'autres. On pourrait raisonnablement penser que ces trajectoires sont propices à des dialogues interdisciplinaires ou peut-être à une certaine forme de porosité entre les disciplines. Mais est-ce le cas ? Si oui, quelles sont les formes de ces échanges?

Il nous semble que ces interrogations et intuitions peuvent recevoir des éléments de réponse au travers de l'analyse de la Revue STAPS. La revue créée en 1980 se propose dès le départ d'être le relais de la recherche dans le domaine et insiste sur la nécessaire représentation de tous les domaines de recherche et, plus que cela, se propose de promouvoir l'interdisciplinarité. Cette revendication est encore fréquemment soulignée :

"Il n'est pas question que STAPS renonce à promouvoir l'interdisciplinarité en STAPS et la langue française, puisque c'est sa vocation depuis sa création en 1980 par les directeurs d'UFR STAPS » (Gleyse, 2009).

Mais en dehors de ce type de professions de foi, on peut interroger les façons dont les scientifiques mettent en scène ou non leur travail interdisciplinaire. Le propos ne vise pas un dévoilement qui soulignerait des écarts entre des velléités interdisciplinaires et la réalité du travail scientifique. Cette posture reposerait en effet sur une conception assez naïve de l'écriture d'un article scientifique comme un compte rendu du travail de recherche réalisé (sur ce point, on peut se référer à l'article de Latour et Fabbri, 1977). Elle supposerait aussi que l'on soit capable de décider si un travail est ou non interdisciplinaire. Le projet n'est pas de défendre une posture normative sur ce que devrait être l'interdisciplinarité. Potentiellement, celle-ci peut prendre plusieurs formes et nous chercherons ici à les identifier dans la Revue STAPS en partant du principe que les auteurs ont à cœur de répondre à la vocation rappelée dans les éditoriaux et ainsi de rendre lisible le travail interdisciplinaire. Il s'agit de regarder, au-delà de la rhétorique de la recherche et des préconisations politiques, comment s'inscrit l'interdisciplinarité dans le texte scientifique. En s'intéressant à un domaine pluridisciplinaire et à une revue qui revendique l'interdisciplinarité comme une originalité, notre objectif est de percevoir quelles formes particulières elle peut revêtir.

\section{MÉTHODE}

Notre travail se fonde sur l'analyse de 602 articles de la Revue STAPS de 1980 à 2010. Tous les articles ont été numérisés. L'analyse a été outillée par le logiciel Prospéro qui a permis de mener l'enquête dans ce large corpus, pour identifier systématiquement les façons d'exprimer des aspects d'une recherche interdisciplinaire. Notre travail a d'abord consisté 1) à identifier les différentes manières de parler d'interdisciplinarité dans les textes, et 2) à regarder les inscriptions disciplinaires des articles.

Pour le premier point, nous avons repéré les textes évoquant explicitement la question de l'interdisciplinarité en construisant un "concept ${ }^{2}$ regroupant l'ensemble des manières possibles de parler d'une forme

1 Le recueil des articles a été réalisé au moyen du site de l'AFRAPS et du CAIRN. Les articles de l'année 2000 étant manquants, Gilles Bui-Xuan nous les a transmis sous forme numérisée. Nous l'en remercions. Ce travail a fait l'objet d'une présentation partielle lors de la Biennale de l'AFRAPS en 2011. En outre il s'insère dans un projet ANR nommé Trascinter portant sur le travail interdisciplinaire dans les sciences du sport. Nous nous centrerons dans cet article plus proprement sur les STAPS en tant qu'espace institutionnel et scientifique. 2 Notre logiciel propose trois sortes de concepts, il s'agit ici d'un « être fictif » qui regroupe tous les synonymes et formes d'écriture d'un mot donné. 
d'interdisciplinarité entendue au sens large (conformément à notre posture initiale et non contraignante) : interdisciplinarité, pluridisciplinarité, "juxtadisciplinarité », multidisciplinarité, transdisciplinarité... À partir de ce concept (appelé Être Fictif dans le logiciel) nous avons pu faire un corpus de textes spécifiques dont la caractéristique est de comprendre pour chacun des textes au moins une apparition d'un des mots liés à Interdisciplinarité (voir ci-dessus). Nous avons ensuite pu analyser ce corpus spécifique (60 textes) pour analyser la manière dont est convoqué le terme et comment l'auteur le travaille. Il s'est agi à ce moment-là d'une analyse de contenu classique.

Pour le deuxième point, le repérage disciplinaire nous permet d'envisager des textes qui croiseraient plusieurs disciplines ou d'envisager comment s'effectueraient des métissages disciplinaires. Pour ce faire nous avons procédé de deux manières conjointes et croisées. Nous avons lu les textes et repéré les marquages disciplinaires par plusieurs éléments : l'explicitation d'une marque de référence (par exemple, un univers professionnel disciplinaire, une mobilisation de concepts, de méthodologies, d'auteurs clairement identifiables...) ou allusion explicite à un champ disciplinaire. Dans le cas suivant on peut, par exemple, observer la répétition d'auteurs et de termes typiquement sociologiques:

«Cette perspective a pour cible les travaux de P. BOURDIEU et la notion d'habitus qui y est développée, notion selon laquelle les classes tendent à se reproduire, classes composées alors selon R. BOUDON, d'individus qui ne sont que de simples exécutants de rôles définis au préalable (p. 241)» (Le Pogam, 1980).

L'allusion explicite à un champ disciplinaire se fait, elle, souvent par la marque de l'intégration du chercheur dans une communauté scientifique précise comme par exemple :

"Comme de coutume, les humbles éducateurs ne suscitent guère la curiosité de l'historien et leur action discrète ne laisse guère de traces " (Arnaud, 1991).

Une deuxième piste a consisté à repérer, outre les repérages disciplinaires évidents (comme dans les titres par exemple: M. Lévêque, «Psychologie du sport : à propos d'une demande d'intervention ", Revue STAPS, $n^{\circ}$ 39, 1996, pp. 83-91), des concepts centraux typiques d'un mode d'approche. Ainsi, un article parlant d'habitus, de champ, de dispositions et de violence symbolique a-t-il toutes les chances d'être un article de sociologie. De la même manière un article évoquant des concepts comme ceux d'attribution causale, de but d'accomplissement, d'estime de soi, de modèle transactionnel... donne un indice fort de l'inscription du travail dans le domaine de la psychologie comme par exemple :

« La distinction entre les deux buts d'accomplissement et la prise en compte des variables cognitives qui leur sont liées (habileté et difficulté normative perçues pour le but de compétition, et probabilité subjective de réussir pour le but de maitrise) semblent plus heuristiques que les modèles antérieurs; cette expérience le confirme, au moins avec une population de garçons » (Sarrazin, 1995).

Ce travail a débouché sur la constitution de lexiques disciplinaires ${ }^{3}$ puisant leur source dans la lecture des articles mais aussi dans un travail sur les concepts ou notions caractéristiques des disciplines au moyen de l'analyse d'ouvrages et de manuels spécialisés dans les domaines scientifiques couplés avec des entretiens de spécialistes des domaines $(n=15)^{4}$.

3 Quelques extraits du lexique Physiologie: acide lactique, acides aminés, acides gras, actine, adénosine diphosphate, adipocytes, ADN, alvéole, AMP cyclique, anabolisant, aorte, aptitudes aérobie, atropine, catécholamines, collagène, consommation maximale d'oxygène, créatine, cycle de Kreps, dette d'oxygène, électrocardiogramme... (le lexique Physiologie comporte 328 représentants).

4 Les critères de choix pour les entretiens ont consisté à interroger des professeurs spécialistes reconnus d'une discipline scientifique dans le domaine des STAPS. Nous avons balayé la pluralité des disciplines représentées dans le domaine. Les entretiens seront notés de E1 à E15. La grille d'entretien prévue pour une recherche plus large sur l'interdisciplinarité comportait de nombreux items mais un de ceux-ci consistait à demander aux chercheurs leur discipline de travail et les notions centrales de leurs travaux scientifiques ainsi que leur inscription ou non dans des travaux interdisciplinaires et les problèmes qu'elle pose. 
Ces derniers ont permis de confirmer ou d'affiner des catégories de notions regroupées de façon non ambigüe/explicite dans une discipline. Ainsi, par exemple, des concepts comme «représentation » ou «stratégie ", utilisés par plusieurs disciplines ne figurent pas dans les Catégories construites. Finalement, nous avons constitué des Catégories par champ disciplinaire (Psychologie, Sociologie, Physiologie...), nous avons pu grâce aux fonctions du logiciel, soit isoler un corpus spécifique par champ disciplinaire, soit constituer des corpus de textes mobilisant les concepts de plusieurs champs disciplinaires (croisement entre psychologie et sociologie, par exemple...).

Nous avons par ailleurs recueilli par l'intermédiaire du moteur de recherche FACTIVA les textes des journaux de la presse française comprenant le mot « interdisciplinarité » et les termes associés (pluridisciplinarité, transdisciplinarité) de 2008 à 2011. Nous avons obtenu un corpus de 17 textes. Celui-ci n'a pas fait l'objet d'une analyse systématique comme pour la
Revue STAPS mais a été utilisé pour contextualiser nos propos.

\section{RÉSULTATS}

\subsection{L'interdisciplinarité :} un objet/une question souvent discuté

Sur 602 articles nous avons repéré 60 articles évoquant l'interdisciplinarité (c'està-dire les mots regroupés dans l'Être fictif Interdisciplinarité, voir ci-dessus) ; parmi eux se trouvent aussi des éditoriaux. L'évolution temporelle de ceux-ci, représentée dans le graphique suivant, montre la constance de cette préoccupation mais souligne aussi l'actualité récente de la thématique. L'augmentation des articles évoquant la question de l'interdisciplinarité dans la période récente est surtout le fait des éditoriaux.

Les récentes évolutions de la politique de la recherche et ses mesures évaluatives imposant la constitution d'une liste de revues de

Tableau 1. Évolution temporelle des articles évoquant explicitement l'interdisciplinarité par rapport au corpus global

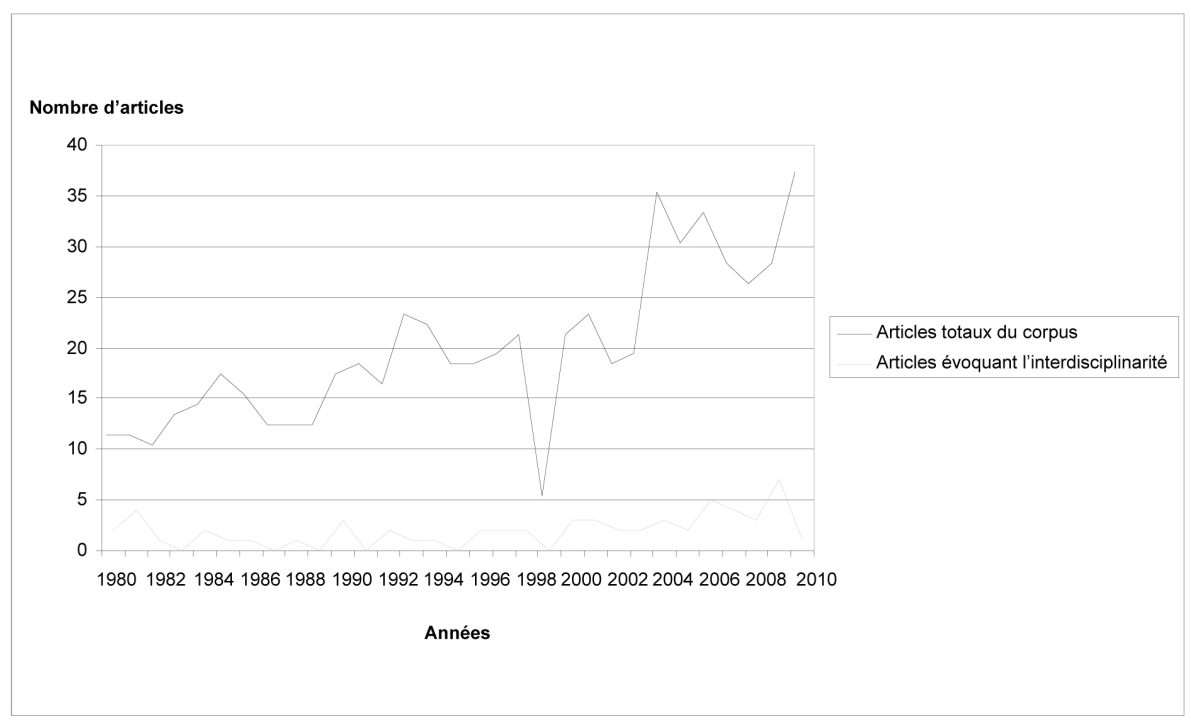

En pointillé, on trouve les articles évoquant explicitement l'interdisciplinarité (mots associés dans l'Être fictif Interdisciplinarité) et en trait plein la totalité des articles sur la période considérée. 
référence ont mis en difficulté certaines de celles-ci et ont pointé la difficile reconnaissance scientifique de l'interdisciplinarité. La Revue STAPS a été particulièrement touchée par ces procédures et son absence de la liste des revues indexées par l'AERES en STAPS a suscité de nombreuses réactions visibles dans le corpus. Les propos tenus dans les différents éditoriaux en 2009 témoignent de cette position difficile et la lient au positionnement interdisciplinaire de la revue :

«En effet, si les STAPS sont assimilables à de la physiologie Stricto sensu à de la psychologie, à de la sociologie, à des sciences de l'éducation, à de l'anthropologie, à de la biomécanique, à de l'histoire et n'ont aucune revue spécifique. À quoi servent les STAPS ? Les STAPS au contraire par le modèle d'interdisciplinarité et de pluridisciplinarité qu'elles offrent aux auteurs permettent la solidification du champ. » (Gleyse, 2009).

La tension résultant de l'attitude qui consiste à encourager l'interdisciplinarité comme mode de travail scientifique innovant et à négliger la reconnaissance scientifique de revues interdisciplinaires est largement ressentie dans la communauté scientifique, nous reviendrons sur ce point.

Nonobstant cet accroissement récent des discussions sur l'interdisciplinarité dans les éditoriaux, elle reste de manière générale un thème cher à la revue et a largement été discutée dans ses colonnes depuis sa création. Cette discussion a pris plusieurs formes.

Parmi les 60 textes recueillis certains (six d'entre eux) ne font qu'une allusion superficielle à la notion, essentiellement au moment du cadrage théorique et souvent pour insister sur la pluralité des travaux portant sur leur objet de recherche, comme l'article de Chantelat faisant un bilan de la littérature socio-économique sur le sport et évoquant des travaux interdisciplinaires :

«Dans un second temps, le regroupement des textes croise deux critères permettant de rendre compte des grandes orientations développées au sein des textes : (...) l'orientation disciplinaire de l'explication en dernière instance du phénomène sportif : économie (politique, normative...), sociologie (économique, critique...), sociologie et économie, voire interdisciplinarité plus large » (Chantelat, 1996).

D'autres articles se positionnent de manière plus prononcée sur l'interdisciplinarité. Certains la revendiquent comme mode privilégié du travail scientifique. Ainsi l'article de Prévost insiste-t-il sur l'intérêt de la démarche interdisciplinaire donnant tout son intérêt à l'analyse épistémologique :

« L'épistémologue en appelle à la pluridisciplinarité, à l'interdisciplinarité, à la transversalité du savoir » (Prévost, 1988).

Si la démarche interdisciplinaire permet de défendre une conception de l'activité scientifique, elle est convoquée aussi pour mieux éclairer certains objets :

«Les progrès viendront d'échanges interdisciplinaires qui seuls permettront d'échapper aux illusions liées aux centrations uniques, de relativiser certaines positions, d'unifier les savoirs "régionaux" pour aboutir à une vision globale, sinon totale, nécessairement transdisciplinaire, des phénomènes agressifs » (Pfister, 1980).

Cette revendication de la nécessité de l'interdisciplinarité dans le travail scientifique s'appuie sur trois arguments essentiels.

Le premier est lié à une forme de défense territoriale. Il s'agit alors pour le chercheur de défendre un territoire qu'il peut sentir menacé et d'affirmer l'intérêt de son approche scientifique. L'interdisciplinarité est alors un argument de mise en valeur scientifique, d'originalité. C'est notamment l'enjeu de l'article de Prévost (cf. supra). L'épistémologie n'est pas, dans le domaine des sciences du sport, une discipline bien installée bien qu'elle soit assez bien représentée dans la Revue STAPS. Elle reste marginale, aussi l'auteur éprouve-t-il le besoin d'affirmer que l'interdisciplinarité permet à l'épistémologie de se trouver dans une 
position avantageuse et donne l'assurance de son intérêt.

On retrouve dans l'article cité un deuxième enjeu lié à la revendication de l'interdisciplinarité : la productivité ou la créativité scientifique déjà évoquées par Vinck (2000) :

"C'est un fait, administratif et non épistémologique, qui veut qu'en France la "transversalité" et l'interdisciplinarité d'un chercheur "border-line" le dessert, alors que c'est ce chercheur qui fait le mieux progresser la science actuelle» (Prévost, 1988).

En effet, la transversalité dont parle l'auteur fait « mieux progresser la science... » et permet de produire de nouveaux savoirs ou de questionner de manière nouvelle des sujets déjà bien frayés.

L'interdisciplinarité est aussi convoquée parce qu'elle permet de répondre à une demande sociale et à la nécessité de faire face à des problèmes de plus en plus complexes dépassant le plus souvent les limites mêmes des frontières disciplinaires. Ainsi, par exemple, la nécessité de comprendre les mécanismes scolaires et notamment ceux de l'apprentissage pour rendre compte des problèmes qui s'y attachent amènent forcément, d'après certains auteurs, à une analyse interdisciplinaire sous peine de ne pouvoir rendre compte de la complexité des phénomènes :

«Dans le domaine de l'éducation physique en particulier, l'analyse des stratégies d'apprentissage en contexte scolaire authentique suppose une approche pluridisciplinaire s'attelant à la complexité » (Ching Wei Chang, 2006).

On retrouve les mêmes arguments dans les travaux sur l'entrainement.

Il arrive aussi, plus rarement, que l'interdisciplinarité soit remise en question. Nous avons pu repérer un texte qui questionne un usage un peu trop facile de la notion :

«Toute pensée préoccupée par son intervention sur l'objet a bien souvent tendance à être satisfaite lorsqu'elle parvient à désigner un phénomène par un mot savant. En se laissant séduire par des discours ou des littératures qui utilisent le pouvoir magique des mots techniques et qui entretiennent parfois des relations privilégiées avec les théories les plus modernes - leur actualité les rendant $a$ priori plus vraisemblables elle évite la confrontation directe et personnelle avec la réalité, et en quelque sorte cherche à échapper aux contraintes et aux efforts de la pratique. La pensée naturelle sous-jacente affectionne alors les jeux avec les homonymes et les synonymes et prétend accéder à l'interdisciplinarité en utilisant par exemple des associations, par simple contiguité spatiale, de notions ou même de concepts issus de cadres théoriques distincts » (Bruant \& Corrand, 1980).

Le contexte de publication du texte cité (1980) est important et rend bien compte de l'interrogation épistémologique d'une discipline qui a encore tout à construire dans le domaine de la recherche (Collinet, 2003). Notons que nous retrouvons dans ce texte les questions de défense territoriale puisque les propos des auteurs visent clairement à fustiger des approches se revendiquant interdisciplinaires comme celles de la didactique. Dans le jeu complexe des positions disciplinaires, l'interdisciplinarité peut être tantôt mobilisée comme argument défensif de l'intérêt d'une approche, tantôt comme point faible de celleci. Les STAPS sont depuis leur naissance traversée par ces débats.

Enfin certains articles prennent l'interdisciplinarité des STAPS comme objet d'étude. Deux auteurs le font avec des démarches différentes, comme Jarnet pour l'épistémologie (ou P. Terral pour la sociologie) :

«Qu'en est-il ? Y a-t-il ou non un objet spécifique, des objets spécifiques ou bien des traditions de recherches et des paradigmes particuliers aux STAPS? Les STAPS sont-elles une science, un savoir pluridisciplinaire, des rationalités hétérogènes ou bien une science plurielle? Comment établir une certaine identité et unité des STAPS? Dans cet article, le terme épistémologie sera pris à la fois au sens de théorie de la connaissance et de critique de la science» (Jarnet, 2004). 
L'enjeu des travaux est de proposer un questionnement sur les STAPS en tant que discipline universitaire, leur organisation, leur fonctionnement et les modes de travail scientifique à l'œuvre dans ce domaine par nature pluridisciplinaire. L'interdisciplinarité est alors un objet d'étude comme les autres, particulièrement pertinent quand on s'intéresse au champ des sciences du sport et de son principal support institutionnel : les STAPS. Si elle apparaît comme objet d'étude lorsque l'on s'intéresse aux STAPS, c'est parce qu'elle est au cœur de leur constitution et qu'en même temps elle pose un certain nombre de problèmes qui alimentent les controverses. Ainsi, les rapports entre les disciplines en STAPS sont-ils complexes. On a pu déjà montrer (Collinet, 2003 ; Terral, 2003) combien les positions pouvaient diverger : entre une conception encourageant une forme de juxtaposition des disciplines qui tendent à se développer en lien étroit avec leur discipline-mère dans une sorte de séparatisme, une autre qui invite les disciplines à collaborer et à échanger tout en restant dans leur spécificité épistémologique (position qu'a défendue par exemple Vigarello; voir notamment son entretien dans l'ouvrage de Collinet, 2001), et enfin une dernière qui envisage une véritable transdisciplinarité entre des modèles qui, en se métissant, produisent une forme nouvelle de connaissance (comme la praxéologie motrice de Parlebas).

\subsection{L'interdisciplinarité : un mode de travail peu présent ?}

Nous avons dénombré neuf articles proposant un travail explicitement interdisciplinaire ${ }^{5}$, ce qui reste très marginal. Ces textes présentent la particularité de revendiquer une approche globale pour traiter des objets complexes.
Deux d'entre eux se questionnent sur l'action motrice et tentent de l'envisager dans sa complexité. Ainsi un texte de 1984 porte-t-il sur l'analyse des jeux collectifs (Menaut, 1984). Le projet de l'auteur est de "mettre en évidence une structure spécifique, "une structure fondamentale" (Dietrich, 1968), coordonnant les aspects dualistique et communicationnel en un système unique " dans les jeux collectifs. Dans le même esprit l'article de Courtay, Heyraud et Roncin propose une réflexion sur l'action motrice «à la fois objet socioculturel et objet biophysique; elle se trouve ainsi au cœur d'une problématique à la fois philosophique et scientifique, entre les sciences sociales d'une part et les sciences de la nature et de la vie d'autre part". Il est intéressant de noter que ces auteurs citent souvent Parlebas dont la posture sur l'action motrice invite à la considérer sous les feux croisés d'approches scientifiques plurielles. On retrouve dans les articles cités cette volonté de rendre compte d'un objet dans toute sa complexité (l'action motrice) sans la réduire à un mode d'analyse scientifique singulier.

D'autres textes (deux articles), dans le même ordre d'idées mais sur des objets différents, s'inscrivent dans le projet de développer une approche systémique englobante ${ }^{6}$. Un texte s'intéresse à la formation : "Les pratiques et les études ponctuelles ou périodiques qui se développent dans le cadre de la formation des adultes, doivent être situées dans une approche globale, de type systémique » (Briglia \& Pithon, 1984) l'autre à l'olympisme : « Cette approche repose sur la science des systèmes - la systémique -, une science conçue pour étudier les "objets complexes" (Chappelet, 1993). Cette notion de complexité caractérise la manière dont les auteurs vont justifier leur recours à l'interdisciplinarité.

5 Annoncé comme tel par les auteurs. Nous avons repéré les textes qui évoquaient les termes liés à l'interdisciplinarité (cf. cadre méthodologique).

6 L'approche systémique chez ces auteurs se fonde sur la mobilisation d'outils scientifiques empruntés à différents champs scientifiques. Néanmoins, cette équivalence interdisciplinarité - approche systémique peut être discutée. D'aucuns considèrent cette dernière comme un paradigme à part entière en sciences sociales. 
On retrouve cette problématique dans les autres textes posant un problème pratique lié à l'entraînement ou la performance. Ainsi les auteurs de l'extrait suivant déplorent-ils la prédominance d'approches scientifiques uniques dans l'analyse de la performance et présententils ensuite un mode d'analyse pluriel de cet objet :

«Dans l'arsenal des moyens mis en œuvre, il est remarquable que la tendance actuelle est de donner la primauté à des références essentiellement bioénergétiques. Ainsi, les exercices constituant le contenu de l'entraînement sont-ils répertoriés en aérobie lactique ou alactique (endurance ou résistance en termes de terrain). Pareille attitude prévaut en dépit des développements considérables d'autres approches de l'entrainement. Non seulement, les aspects sensoriels, perceptifs, sensori-moteurs, comportementaux, psycho-sociaux, voire même les contingences biomécaniques de l'activité physique sont tenues pour des déterminismes secondaires des prestations sportives, mais tout se passe presque comme si l'athlète assimilé à un moteur transformant l'énergie chimique musculaire en travail mécanique seul le versant thermodynamique des phénomènes dont il est le siège était pris en compte » (Bruant \& Thys, 1990).

L'objectif d'optimisation de la performance semble imposer d'avoir recours à l'interdisciplinarité :

"Notre second objectif est de définir les bases d'une réflexion qui se veut pluridisciplinaire, c'est-à-dire qui s'efforce d'aller au-delà de la simple juxtaposition des divers discours scientifiques sur le comportement moteur. Cependant, adopter une approche pluridisciplinaire ne va pas de soi, tant les contenus scientifiques sont devenus spécifiques et relèvent de logiques propres. C'est pourquoi, plutôt que de tenter une analyse des différents niveaux d'organisation de la motricité, notre réflexion portera sur les interactions entre les systèmes biologiques sollicités lors de la réalisation d'une tâche, à partir de l'étude de leurs propriétés fonctionnelles essentielles » (Durand, 1992).
Ces textes présentent explicitement un mode d'approche interdisciplinaire. Il est possible de les associer avec ceux qui se proposent de s'inscrire dans deux cadres disciplinaires sans pour autant revendiquer une forme explicite d'interdisciplinarité. Ces articles se différencient des neuf premiers par le fait qu'ils ne mentionnent pas explicitement (comme une revendication) le travail interdisciplinaire. Une trentaine d'articles sont concernés. Les croisements disciplinaires les plus fréquents ont lieu entre la sociologie et l'histoire comme par exemple le travail de Lê-Germain et Leca (2005) sur les conduites dopantes. La problématique de nature sociologique est envisagée sur une période historique allant de 1965 à 1999 : « (...). Car notre souci est moins de décrire des listes, des produits et procédés, illégaux le plus souvent que de montrer que ces conduites spécifiques fondent une sous-culture sportive, celle qui organise certains groupes de coureurs cyclistes. » On retrouve ce type de démarche dans certains travaux de Terret (2003).

Dans cet ensemble de nombreux textes se situent aussi dans une interface entre sciences de l'intervention ou didactique (dont on rappelle que ses tenants la définissent comme une discipline scientifique) et psychologie, comme par exemple dans le travail de Lafont (2002). Cette hybridation (au sens métaphorique de croisement) pose évidemment le problème du statut épistémologique du domaine de l'intervention (ou de la didactique) en tant que discipline scientifique dont la posture oscille entre reconnaissance d'une forme d'interdisciplinarité sur des objets spécifiques (liés à l'enseignement) et spécificité disciplinaire (reconnaissance d'une discipline scientifique à part entière) comme en témoigne cet extrait d'entretien : «Je fais de la didactique mais je n'ai pas envie de me positionner dans un champ scientifique. J'utilise les concepts qui me servent pour analyser les problèmes de terrain, que ce soit emprunté à la psychologie, à la sociologie ou à la physiologie quand c'est nécessaire. Après je m'en sers pour apporter des réponses aux questions que je me 
pose sur les problèmes concrets de l'enseignement » (E 15).

Enfin, certains mélanges sont plus marginaux (c'est-à-dire moins nombreux) comme ceux associant physiologie et sociologie, comme dans l'étude des relations entre APS, alimentation et intégration sociale chez des femmes incarcérées (Garnier, Mejean \& Minotti, 1990), ou ceux mêlant psychologie et sociologie autour d'une étude sur le dopage (Brissonneau \& Bui-Xuan-Picchedda, 2005).

Finalement, une quarantaine de textes sur 602 proposent un travail interdisciplinaire (dont neuf le revendiquent explicitement). Si l'on se fie au contenu des articles, on pourrait penser que le travail interdisciplinaire pose finalement plus de problèmes qu'il n'apporte d'éléments positifs à la recherche. Certains de ces problèmes ont été soulignés dans les discours sur l'interdisciplinarité, dont on a vu que tout un versant incitatif et laudatif était relayé à la fois dans la presse et dans les écrits des chercheurs eux-mêmes. Ainsi peut-on aussi remarquer que le manque de moyens et les problèmes de financement sont un frein au travail interdisciplinaire : "Depuis quelques années, le ministère nous incitait à faire de la multidisciplinarité mais il n'y avait aucun moyen de le concrétiser! » (Les Échos, 8 février 2006). Par ailleurs, si l'on regarde, par exemple, les commentaires des évaluations de l'AERES des laboratoires STAPS on peut voir que la disciplinarité reste le critère d'excellence de publication scientifique, renforcé par le classement des revues $^{7}$ fait par cet organisme. Ainsi peut-on lire dans les recommandations de groupes d'experts-évaluateurs qu'il faut «trouver un équilibre entre production scientifique de bon niveau mettant en exergue les compétences disciplinaires fortes d'une part et les compétences interdisciplinaires fortes d'autre part » (Document AERES $1^{\text {er }}$ mai 2008); " $\mathrm{A}$ cet égard, en se référant aux critères de l'AERES et en tenant compte du fait que, dans les domaines concernés par les formations pluridisciplinaires, les revues dites de rang A sont peu nombreuses alors que les supports demandeurs de résultats de recherche appliquée sont au contraire abondants » (Document AERES $1^{\mathrm{er}}$ mars 2009). Ces arguments sont alors mobilisés pour inciter les chercheurs à publier dans des revues «payantes» (pour reprendre les termes des interviewés). La Revue STAPS souffre, en tout cas aujourd'hui, particulièrement du manque de reconnaissance scientifique de l'interdisciplinarité, comme nous avons pu le souligner; ses éditoriaux en portent d'ailleurs la trace très nette. Même si des investigations approfondies seraient nécessaires pour préciser les processus à l'œuvre, on peut penser que la difficulté de publication des travaux interdisciplinaires couplée aux formes de publication encouragées par les instances évaluatives nationales constitue un frein au développement de telles recherches.

En dehors de ces contraintes institutionnelles, on peut interroger le coût cognitif et/ ou humain du travail interdisciplinaire : mầtrise de concepts, de méthodes différentes, coordination difficile avec d'autres chercheurs. Ces contraintes cognitives et pragmatiques sont soulignées par les chercheurs que nous avons interrogés dans notre enquête :

"Le problème de l'utilisation des concepts
sociologiques dans mon travail d'historien,
c'est que les sociologues me tombent tou-
jours dessus en me disant que je ne les maî-
trise pas assez, qu'ils sont dépassés, discutés,

7 Si nous prenons par exemple la liste des revues AERES proposées dans le domaine des STAPS, 250 revues sur 456 font mention explicite d'une discipline soit à peu près $55 \%$ (nous n'avons pas retenu dans ce comptage les disciplines elles-mêmes pluridisciplinaires comme la communication ou le management, de même que nous n'avons pas compté les revues relatives au domaine de l'éducation). Dans ces disciplines nous retrouvons essentiellement l'histoire, la sociologie, l'économie, la psychologie, la physiologie, la biomécanique, les neurosciences et la médecine. À cet ensemble de revues disciplinaires nous pouvons ajouter un certain nombre de revues dont le titre ne porte pas mention explicitement d'une discipline tout en étant cependant orientées vers une seule discipline, comme la revue Stadion pour l'histoire par exemple ; nous pouvons ainsi comptabiliser environ $65 \%$ de revues disciplinaires. Parmi celles-ci, 108 revues sont classées en premier rang, ce qui représente $67 \%$ des revues de premier rang de la liste. 
remis en question... et finalement bien que je trouve cela heuristique, je me sens souvent trop juste pour les faire travailler sur ma période historique de manière vraiment pertinente» (E 13).

«En histoire contemporaine on nous incite à nous faire qualifier en sociologie ou en sciences politiques et d'avoir recours à une certaine interdisciplinarité dans nos modèles et nos concepts. En fait, on essaie de coller un peu artificiellement quelques concepts de ces domaines en regardant par exemple ce qui se fait dans les revues disciplinaires dans lesquelles on veut publier mais ça reste superficiel» (E 14).

\subsection{Des concepts comme marques de porosité disciplinaire}

Faut-il en conclure que la part de l'interdisciplinarité est particulièrement faible dans une revue qui n'a de cesse de l'afficher et d'en faire sa spécificité? Ce serait aller bien vite ou en tout cas ignorer que des formes moins lisibles, qui échappent aux lexicomètres et autres outils bibliométriques, peuvent être présentes.

D'une part, nous l'avons vu, parce qu'il existe des activités scientifiques, à l'instar de la didactique (mais c'est également vrai pour la recherche en management) qui revendiquent à la fois une spécificité disciplinaire et un travail interdisciplinaire.

D'autre part, parce que l'on peut considérer qu'une manifestation de la présence d'interdisciplinarité peut se lire dans des textes dans lesquels les auteurs de la revue mixent des concepts relevant de différentes disciplines. En d'autres termes, ils se trouveraient engagés dans des formes d'interdisciplinarité sans le revendiquer explicitement. Pour mettre à l'épreuve cette hypothèse, l'analyse a porté sur les rapprochements de la psychologie et de la sociologie. La connaissance de ces deux domaines disciplinaires et de la psychologie sociale qui se présente souvent comme articulant les deux regards, a permis d'épurer les lexiques disciplinaires. Le logiciel étant conçu comme une machine permettant de faire des expériences sur les textes, il a été procédé à plusieurs tentatives visant à interroger la possibilité d'articulations disciplinaires a priori inattendues.

Tout d'abord, nous avons construit des catégories assez larges renvoyant à chacune des deux disciplines. Nous avons pour cela retiré les termes ambigus comme « champ » qui peut renvoyer à la notion sociologique mais aussi à des champs de vision, ou comme "reproduction » qui pointe un lexique bourdieusien et une tâche motrice ou encore le concept de «norme » qui désigne à la fois un ordre social et une grandeur statistique, mais nous avons gardé des représentants qui restent travaillés de façon plus ou moins semblables dans les deux disciplines à l'instar de "représentation sociale », « interaction », « conflits ». Nous parvenons ainsi à identifier 414 articles mobilisant au moins une fois un représentant des deux catégories. L'objectif consiste à repérer les façons qu'ont les auteurs-acteurs d'articuler des considérations dans deux disciplines.

Dans un second temps, nous avons durci nos critères pour restreindre ce sous-corpus et ainsi porter l'analyse de façon plus fine sur un ensemble de 345 textes engageant plus directement des lexiques émanant des deux disciplines.

Enfin, nous avons travaillé de façon manuelle - soit sans le logiciel - pour isoler respectivement deux sous-corpus d'articles qui relèvent sans trop d'ambiguités des deux champs disciplinaires. Cette opération qui a vocation à terme à être automatisée, s'est effectuée en croisant non seulement les lexiques, mais aussi des données bibliométriques ainsi qu'une enquête sur les auteurs, leurs profils et leurs publications.

Dans ces sous-corpus, nous avons cherché à repérer sur quoi portaient ces textes "pluridisciplinaires ", les façons dont se convoquent les concepts d'une autre discipline et le statut de ces mobilisations dans l'argumentation.

À la lecture, nous avons noté combien de textes portaient, de près ou de loin, sur la 
didactique, l'EPS, les relations enseignant-enseigné. Pour mettre à l'épreuve cette hypothèse, nous avons décidé d'isoler les articles sur la thématique de l'école (la pluridisciplinarité évoquée entre l'EPS et les autres disciplines scolaires nous éloignait de notre objet). Cette opération s'accompagne de la disparition de nombreux textes puisque seuls 52 documents résistent à ces nouveaux critères, $60 \mathrm{si}$ on s'intéresse au corpus plus large. La requête sur les deux sous-corpus conduit à affirmer que 81 textes sur les 99 du corpus «psychologie » concernent des questions éducatives ou scolaires quand 75 sur 98 présentent la même propriété dans le corpus «sociologie ». Ces considérations invitent à penser que les questions d'enseignement génèrent la convocation de deux lexiques disciplinaires (au moins). Nous avons pu montrer par ailleurs que la didactique se positionnait comme un propos puisant dans ces deux disciplines. Nous retrouvons ici une des caractéristiques des STAPS déjà soulignée dans de nombreux travaux (Terral, 2003; Terral \& Collinet, 2007) : son ancrage historique dans une science de l'EPS. Il ne faut en effet pas oublier que la discipline STAPS émane historiquement $\mathrm{du}$ souhait des enseignants d'EPS de produire une science servant les intérêts de ce groupe professionnel.

L'enquête plus approfondie présentée ici vise à interroger la porosité disciplinaire en dehors des chemins très frayés, exigée par un objet comme l'éducation physique et sportive. Pour cela, nous avons isolé les textes ne traitant pas de l'enseignement pour établir les modalités de passage entre les deux champs disciplinaires.

Une partie d'entre eux concerne bien sûr la psychologie sociale mais, si nous durcissons encore les critères, la recherche des notions sociologiques dans un sous-corpus «sciences psychologiques" fait apparaître cinq figures de surgissement du vocabulaire sociologique dans des textes où elles n'étaient pas réellement attendues.

Tableau 2. Cinq formes de surgissement du lexique sociologique dans des textes de psychologie Le tableau présente dans la colonne Figures les cinq figures de surgissement du vocabulaire sociologique dans des textes appartenant à d'autres disciplines.

La colonne Formes précise les modes d'utilisation de ce vocabulaire et la colonne Exemples donne des extraits de textes.

\begin{tabular}{|l|l|l|}
\hline Figures & Formes & Exemples \\
\hline $\begin{array}{l}\text { Contextualisation } \\
\text { de l'objet }\end{array}$ & $\begin{array}{l}\text { Pour présenter, analyser } \\
\text { un objet, on rappelle son } \\
\text { contexte. Le rapport aux } \\
\text { sciences sociales est donc très } \\
\text { généraliste }\end{array}$ & $\begin{array}{l}\text { « .. il est d'autant plus important que l'éducation } \\
\text { des enfants constituant les populations dites } \\
\text { spéciales leur permette de réaliser au maximum } \\
\text { leur potentiel dans ces trois sphères, afin } \\
\text { notamment de favoriser leur intégration sociale } \text { et } \\
\text { leur épanouissement. » (Debu, 1998) }\end{array}$ \\
\hline $\begin{array}{l}\text { Généralisation/ } \\
\text { Parcours de } \\
\text { la littérature } \\
\text { dépassant } \\
\text { les frontières } \\
\text { disciplinaires } \\
\text { classiques }\end{array}$ & $\begin{array}{l}\text { Des inférences sur des } \\
\text { invariants qui s'étendent aux } \\
\text { sciences sociales } \\
\text { Présentation d'un objet } \\
\text { qui dépasse les frontières } \\
\text { disciplinaires }\end{array}$ & $\begin{array}{l}\text { «'expérience du flow serait identique quels } \\
\text { que soient la culture, la classe, le genre et l'âge du } \\
\text { sujet » (Demontrond \& Gaudreau, 2008) } \\
\text { «... que ce soit à propos de la maladie } \\
\text { (HERZLISCH), de la culture (KAES), de l'élève } \\
\text { (GILLY), de l'enfance (CHOMBARD DE LAWE), } \\
\text { tous les auteurs s'accordent pour affirmer que } \\
\text { la pensée en usage fonctionne en réduisant la } \\
\text { complexité du réel pour aboutir à des noyaux } \\
\text { souvent bipolaires » (Le Her, 1988) }\end{array}$ \\
\hline
\end{tabular}




\begin{tabular}{|c|c|c|}
\hline \multirow[t]{2}{*}{$\begin{array}{l}\text { Discussion plus } \\
\text { ou moins critique } \\
\text { entre les deux } \\
\text { disciplines }\end{array}$} & $\begin{array}{l}\text { Analyse sociologique de du } \\
\text { travail du psychologue }\end{array}$ & $\begin{array}{l}\text { "Pendant huit années, de } 1985 \text { à 1992, nous } \\
\text { avons mené auprès des équipes de France de voile } \\
\text { olympique une intervention psychologique sur } \\
\text { le terrain sportif. Indiquons d'emblée que cette } \\
\text { dénomination de notre activité, pour classique } \\
\text { qu'elle soit, ne nous satisfait guère (...). L'appel } \\
\text { au psychologue accorde à sa discipline un crédit } \\
\text { et une légitimité appréciables : participer au } \\
\text { processus de production. Être convié dans le cercle } \\
\text { restreint des disciplines susceptibles de soutenir } \\
\text { l'amélioration de la performance affermit la } \\
\text { spécificité d'un savoir. Mais cette reconnaissance } \\
\text { a pour corollaire de lui imposer des visées très } \\
\text { appliquées, voire de le transformer en "savoir } \\
\text { asservi" » (Lévêque, 1996) }\end{array}$ \\
\hline & $\begin{array}{l}\text { Défense de la posture } \\
\text { psychologique contre } \\
\text { l'approche sociologique }\end{array}$ & $\begin{array}{l}\text { «Les individus se réfèrent davantage à des } \\
\text { standards personnels de réalisation qu'à des } \\
\text { normes sociales. (..) } \\
\text { Une conception générale des systèmes } \\
\text { biologiques, et plus particulièrement l'approche } \\
\text { psychobiologique des comportements moteurs, } \\
\text { dépasse actuellement l'étude des conduites } \\
\text { humaines qui a très longtemps essayé de définir } \\
\text { les normes d'une éthique de la vie individuelle en } \\
\text { relation étroite avec la vie sociale » (Brunel, 1996) }\end{array}$ \\
\hline $\begin{array}{l}\text { Lexique commun } \\
\text { mais usages } \\
\text { différents }\end{array}$ & $\begin{array}{l}\text { Moins que la polysémie } \\
\text { usuelle, il s'agit de notions } \\
\text { travaillées différemment par } \\
\text { les deux disciplines }\end{array}$ & $\begin{array}{l}\text { "Rien ne nous permet d'affirmer que lorsqu'il } \\
\text { effectue les reproductions de la norme il } \\
\text { ne continue pas à utiliser ce qu'il a retenu } \\
\text { visuellement du modèle présenté, donc à } \\
\text { fonctionner sur la base d'un transfert intermodal. } \\
\text { Le discours des sujets sur leur stratégie de } \\
\text { reproduction de la norme, une fois l'essai terminé, } \\
\text { peut laisser penser qu'il n'en est rien » } \\
\text { (Cadopi, 1988). }\end{array}$ \\
\hline $\begin{array}{l}\text { Rapprochement } \\
\text { disciplinaire }\end{array}$ & $\begin{array}{l}\text { Un travail sur les tensions et } \\
\text { les rapprochements entre les } \\
\text { domaines disciplinaires }\end{array}$ & $\begin{array}{l}\text { "Dynamisme motivationnel et perturbation } \\
\text { du système cognitivo-perceptivo-moteur ne } \\
\text { risquent-ils pas d'évacuer d'autres réalités liées à } \\
\text { l'affrontement de deux équipes de sport collectif ? } \\
\text { Si l'on introduit l'idée d'une interaction à la fois } \\
\text { tactique et sociale, c'est pour mieux insister sur } \\
\text { l'inévitable insertion des conduites du joueur dans } \\
\text { ce que l'on appelle une symbolique sociale" } \\
\text { (Rey, 1992). }\end{array}$ \\
\hline
\end{tabular}

Avant d'analyser plus avant ce que ces figures disent de l'interdisciplinarité, nous avons réalisé symétriquement le même type de travail. Dans un corpus d'articles affirmant un ancrage fort en sciences sociales, nous avons exclu selon les mêmes critères les textes touchant précisément à des questions d'EPS. Comme précédemment, le but de cette opération consiste à fixer des critères assez durs pour identifier les formes non envisagées jusqu'alors de présence de l'interdisciplinarité. Ainsi, avons-nous porté l'attention plus spécifiquement sur 38 textes de sciences sociales, ne traitant pas de l'EPS, pour repérer les façons dont peut être mobilisé le lexique psychologique. Nous repérons ainsi quatre figures d'apparition du lexique psychologique en sciences sociales : 
Tableau 3. Quatre formes d'apparition du lexique psychologique en sciences sociales Le tableau présente dans la colonne Figures les quatre figures de surgissement du vocabulaire psychologique dans des textes de sciences sociales. La colonne Formes précise les modes d'utilisation de ce vocabulaire et la colonne Exemples donne des extraits de textes.

\begin{tabular}{|c|c|c|}
\hline Figures & Formes & Exemples \\
\hline $\begin{array}{l}\text { Une } \\
\text { contextualisation } \\
\text { de l'objet }\end{array}$ & $\begin{array}{l}\text { Convocation d'autres } \\
\text { domaines disciplinaires } \\
\text { pour poser l'originalité ou la } \\
\text { complexité d'un objet }\end{array}$ & $\begin{array}{l}\text { «De la psychologie à l'histoire en passant par la } \\
\text { sociologie, force est en effet de constater la rareté } \\
\text { des études en ce domaine » (Terret, 2004). }\end{array}$ \\
\hline \multirow[b]{2}{*}{$\begin{array}{l}\text { Discussion } \\
\text { critique entre les } \\
\text { deux disciplines }\end{array}$} & Sociologie de la connaissance & $\begin{array}{l}\text { « Nous cherchons ici à montrer que la } \\
\text { construction des savoirs est associée à des } \\
\text { processus cognitifs (questions que l'on se pose, } \\
\text { modes de pensée...), discursifs (vocabulaire, } \\
\text { formes argumentaires liées à des modes de } \\
\text { pensée...) et sociaux (contraintes sociales des } \\
\text { contextes de production et de diffusion des savoirs, } \\
\text { processus de légitimation, de reconnaissance, } \\
\text { voire de domination...) apparaissant bien souvent } \\
\text { comme interdépendants » (Terral, 2003). }\end{array}$ \\
\hline & $\begin{array}{l}\text { Défense de la posture } \\
\text { sociologique contre } \\
\text { l'approche psychologique }\end{array}$ & $\begin{array}{l}\text { Plusieurs travaux (Berger, Luckman, } 1985 \text {; Lahire, } \\
\text { 1995) montrent que la socialisation se réalise au } \\
\text { sein d'institutions au sens large et que la famille } \\
\text { est le premier agent de socialisation (De Singly, } \\
\text { 1995). En effet, "la personnalité de l'enfant, } \\
\text { ses "raisonnements" et ses comportements, ses } \\
\text { actions et réactions, sont insaisissables en dehors } \\
\text { des relations sociales qui se tissent, initialement, } \\
\text { entre lui et les autres membres de la constellation } \\
\text { familiale, dans un univers d'objets liés aux formes } \\
\text { de relations sociales intra familiales " (Croquette, } \\
\text { 2004). }\end{array}$ \\
\hline \multirow[t]{2}{*}{$\begin{array}{l}\text { Lexique commun } \\
\text { mais usages } \\
\text { différents }\end{array}$} & $\begin{array}{l}\text { Usage profane de termes } \\
\text { conceptualisés par la } \\
\text { psychologie }\end{array}$ & $\begin{array}{l}\text { Chacun peut, à sa guise, satisfaire des désirs } \\
\text { différenciés selon sa propre personnalité (faire } \\
\text { de la compétition sportive ou se livrer à une } \\
\text { activité physique hors de cette perspective } \\
\text { institutionnelle) ; mais l'objectif final des loisirs } \\
\text { est d'établir un certain nombre de relations } \\
\text { à soi (même par la médiation des autres) qui } \\
\text { privilégient une valeur susceptible de remettre } \\
\text { en cause le rapport entre l'individu et la société, } \\
\text { leurs droits et leurs devoirs réciproques («leisure } \\
\text { requires a sacrifice », écrit de Grazia). }\end{array}$ \\
\hline & $\begin{array}{l}\text { Notions travaillées } \\
\text { différemment par les deux } \\
\text { disciplines }\end{array}$ & $\begin{array}{l}\text { On comprend alors que la diversité des acteurs } \\
\text { en présence (politico-administratifs : communes, } \\
\text { départements, État, etc. ; associatifs : sport, } \\
\text { culture, quartier), et la logique de pensée propre à } \\
\text { chacun d'eux, induisent une complexification des } \\
\text { relations et posent le problème de la définition et } \\
\text { de la coordination d'une politique sportive (Dulac } \\
\text { 1998). }\end{array}$ \\
\hline $\begin{array}{l}\text { Rapprochement } \\
\text { disciplinaire }\end{array}$ & $\begin{array}{l}\text { Un travail sur les tensions et } \\
\text { les rapprochements entre les } \\
\text { domaines disciplinaires }\end{array}$ & $\begin{array}{l}\text { Mais la notion de schèmes, commune à la } \\
\text { psychologie piagétienne et à la sociologie de } \\
\text { P. BOURDIEU, } \\
\text { semble couvrir des réalités à la fois semblables et } \\
\text { différentes (Le Pogam, 1980). }\end{array}$ \\
\hline
\end{tabular}


Que retenir de ce travail ? Nous avons posé des contraintes fortes pour repérer les modalités d'association moins fréquentes entre deux disciplines en espérant ainsi identifier d'autres formes de l'interdisciplinarité. Les tableaux précédents donnent à lire quelques résultats qu'il convient d'analyser :

- On repère plusieurs revues de littérature qui dépassent les frontières disciplinaires. Il faudrait assurément travailler l'interdisciplinarité dans d'autres domaines que les sciences du sport pour affirmer une originalité mais la lecture régulière de revues ancrées dans l'une ou l'autre des disciplines invite à penser que ce processus de convocation d'autres champs disciplinaires est assez rare.

- La recherche de notions de disciplines voisines dans un champ disciplinaire donné rend visible un lexique commun qui peut être source de malentendus. Ce n'est pas la polysémie de la langue qui est visée ici (des mots comme « champ » ou « reproduction»). Mais des mots, utilisés comme concepts dans les deux disciplines, contraignent certains auteurs à préciser que c'est au sens de la psychologie ou de la sociologie qu'il convient d'entendre le terme utilisé. Implicitement, cette précaution suggère que des malentendus sont possibles, que des étudiants ou des collègues peu formés aux autres disciplines peuvent aux yeux des spécialistes de ces disciplines, se méprendre. Il s'agit là d'une difficulté de l'interdisciplinarité, peu étudiée et sans doute sous-estimée, comme le montrent les travaux sur les fallacies (Szpirglas, 2006) qui prennent au sérieux les quiproquos (mauvais usage des termes lorsqu'ils sont employés dans une autre discipline).

- Des discussions entre disciplines qui engagent les présupposés, les prétentions, les limites de chacune d'entre elles sont également repérables. On peut considérer qu'il s'agit d'un gradient supplémentaire vers des travaux interdisciplinaires puisque la charge envers le réductionnisme supposé de l'autre discipline suppose une lecture attentive de ses productions. S'il fallait assurément mener l'enquête sur d'autres activités scientifiques rassemblés sur un objet (l'éducation, le climat, la communication...), on peut noter la rareté d'échanges écrits qui se préoccupent du bon niveau de description du monde.

- On a identifié quelques textes qui travaillent directement les conditions de rapprochement et de tensions entre les disciplines. Cela n'est pas très commun mais ce type de recherche pointe généralement des travaux historiques, épistémologiques ou appartenant à la sociologie de la connaissance. Le fait que ces travaux soient publiés dans une revue à prétention interdisciplinaire n'est donc pas surprenant puisque la revue accepte des articles historiques et épistémologiques mais on peut considérer que ces textes visent moins un propos centré sur les conditions de la production de la connaissance qu'une volonté de clarification conceptuelle.

\section{CONCLUSION}

Au terme de cette analyse de l'interdisciplinarité telle qu'elle se donne à voir dans les articles de la Revue STAPS dont nous avons rappelé la place dans l'histoire des STAPS, plusieurs constats ont été établis. L'objectif n'était tout d'abord pas de pointer d'éventuels écarts entre les annonces relatives à l'interdisciplinarité et des formes de « réalité ». Pour résumer, on peut dire en effet qu'il n'existe pas une mais des « réalités » de l'interdisciplinarité. Notons au passage que ce point interroge l'évaluation interdisciplinaire du travail scientifique à l'heure où ce mot d'ordre, souvent employé au singulier, se fait de plus en plus présent voire pressant dans divers espaces politiques comme nous avons pu l'évoquer en analysant certaines positions politiques dans la presse ou même des préconisations émanant de l'AERES par exemple. À partir de l'étude de la diffusion scientifique et non comme par exemple a pu le faire Vinck (2000) d'ethnographies de vies de laboratoires, notre enquête donne en effet à voir plusieurs figures de l'interdisciplinarité 
comme autant de « mises en scène » de ce type de travail scientifique.

C'est tout d'abord un mot d'ordre, qui, s'il est présent dans nombre d'injonctions politiques actuelles, l'est également dans les textes scientifiques des chercheurs des sciences du sport ainsi que dans les éditoriaux de la revue étudiée comme nous avons pu le souligner. Il ressort de l'analyse que les chercheurs en sciences du sport mobilisent un usage explicite de l'interdisciplinarité en relation avec plusieurs types de préoccupations que l'on peut situer dans la tension entre l'explicatif et le normatif. Si certains auteurs prennent cette thématique comme objet d'analyse, d'autres s'en font clairement des militants. Outre des velléités de réorientation du travail scientifique, notamment dans un souci de connexion plus directe avec la demande sociale (particulièrement celle touchant aux questions d'éducation physique comme nous avons pu le relever), ces derniers portent des préoccupations de défense territoriale. Celles-ci sont paradigmatiques, comme dans le cas de la didactique par exemple, ou disciplinaires et concerne les STAPS dans leur globalité. Comme nous avons pu le souligner, la réflexion interdisciplinaire est étroitement liée à celle sur la construction de la discipline STAPS.

Notre analyse montre toutefois combien la production de recherches interdisciplinaires n'est pas aisée pour les chercheurs des sciences du sport. Outre les difficultés inhérentes à sa mise en œuvre, nombre d'acteurs relèvent également la difficile reconnaissance institutionnelle de ce type de travaux. Dès lors, à titre d'hypothèse de recherche à nourrir par des analyses complémentaires, il nous semble intéressant de creuser l'idée que les figures de l'interdisciplinarité sont à saisir comme des tensions inhérentes à la double contrainte de « disciplinarité » et d'" interdisciplinarité ». Dit autrement, nous pensons que l'interdisciplinarité pose de fait des questions aux disciplines et induit potentiellement une activité réflexive sur la nature du travail scientifique à produire.
Bien que l'analyse doive être approfondie, c'est ce que nous donnent en partie à voir nos investigations des usages de lexiques psychologiques par les sociologues et inversement. En effet, qu'il s'agisse d'opérations de contextualisation de l'objet de recherche via une autre discipline ou de discussions critiques entre deux domaines de connaissance, l'activité interdisciplinaire engage un travail réflexif sur les façons de faire de la science. Reste à préciser, par l'intermédiaire d'investigations plus approfondies visant notamment l'analyse de plusieurs revues des sciences du sport, la nature de cette activité réflexive en acte et ce qui amène certains chercheurs à la mettre en œuvre.

\section{BibLIOGRAPHIE}

ARnaud, P. (1991). L'éducation physique à Lyon. Réussite et ambiguités d'une politique municipale (1919-1939). STAPS, 55, 7-21.

Boisot, M., HeCKhausen, H. (1972). Discipline et interdisciplinarité, in OCDE (Ed.), L'interdisciplinarité problèmes d'enseignement et de recherche dans les universités.

Briglia J.-P., Pithon, G. (1984). Besoins en formation et participation aux activités de formation des enseignants d'éducation physique et sportive dans l'académie de Montpellier. STAPS, 10, 52-63.

Brissonneau, C., Bui-Xuan-Picchedda, K. (2005). Analyse psychologique et sociologique du dopage. Rationalisation du discours, du mode de vie et de l'entraînement sportif. STAPS, 70, 59-73.

Bruant, G., Corrand, B. (1980). Théorie et pratique: pas de sens interdit, plusieurs itinéraires possibles. STAPS, 1, 3-7.

BRUANT, G., THYS, H. (1990). Des données de la recherche à l'entraînement. Réflexions sur l'application des connaissances scientifiques. STAPS, 22, 57-60.

Chantelat, P. (1996). La littérature socio-économique du sport en France : essai de classification et questionnement épistémologique. STAPS, 41, 55-75.

Chappelet, J.-L. (1993). Approche systémique du phénomène olympique. STAPS, 30, 21-30.

Collinet, C. (2001). EPS et sciences. Paris, PUF.

Collinet C. (2003). La Recherche en STAPS. Les tensions essentielles. Paris, PUF, coll. "Sciences de l'éducation. Section Recherches scientifiques ".

Collinet, C., Payré, S. (2004). La communauté des enseignants-chercheurs en STAPS entre spécificité et 
perméabilité. Travaux et recherches de l'université de $M L V, 165-193$.

DuRAND, M. (1992). L'optimisation de la performance. STAPS, 27, 7-13.

Frodeman, R., Thompson Klein, J., Mitcham, C. (Eds.). The Oxford Handbook of Interdisciplinarity. Oxford, Oxford University Press, 2010.

Garnier, S., Mejean, L., Minomi, C. (1990). Influence des activités physiques et sportives sur l'alimentation des femmes incarcérées. STAPS, 23, 51-56.

GLEYSE, J. (2009). Éditorial. STAPS, 84.

JANTSCH, E. (1972). Vers l'interdisciplinarité et la transdisciplinarité dans l'enseignement et l'innovation, in OCDE (Ed.), Linterdisciplinarité problèmes d'enseignement et de recherche dans les universités.

JARnet, L. (2004). Pour une épistémologie aposterioriste des STAPS. STAPS, 65, 27-41.

KLEIN, J.T. (1990). Interdisplinarity: History, theory and Practice. Michigan, Wayne State University Press.

LAFFoNT, L. (2002). Efficacité comparée de la démonstration explicitée et de l'imitation-modélisation interactive pour l'acquisition d'une séquence dansée chez des adolescentes de 12 à 15 ans. STAPS, 58, 69-79.

Latour, B., Fabbri, P. (1977). Pouvoir et devoir dans un article de science exacte. Actes de la Recherche en Sciences Sociales, 13, 81-95.

LÊ-Germain, E., LECA, R. (2005). Les conduites dopantes fondatrices d'une sous-culture cycliste (1965-1999). STAPS, 70, 109-125.

MaAsen S., Lieven 0. (2006) Transdisciplinarity: a new mode of governing science? Science and Public Policy, 33 (6), 399-410.

Menaut, A. (1984). Validité et limites du modèle opératoire dans le domaine des jeux sportifs collectifs. STAPS, 10, 29-43.

Piaget, J. (1972). L'épistémologie des relations interdisciplinaires, in OCDE (Ed.), Linterdisciplinarité problèmes d'enseignement et de recherche dans les universités.
Pfister, R. (1980). La mesure de l'agressivité. STAPS, 2 , 23-33.

PrÉvost, C.M. (1988). Sciences et techniques des APS : réflexions épistémologiques. STAPS, 17, 7-16.

Prud'homme T., Gingras Y., Couillard A., Terrasson D. (2012). «Les mesures de l'interdiscplinarité. Pratique et attitudes dans un centre de recherche français : l'IRSTEA ». CIRST, 01, http://www.cirst. uqam.ca/Portals/0/docs/note_rech/2012-01.pdf.

SinACEUR, M. A. (1992). "Quelques réflexions sur l'interdisciplinarité ", in Entre savoirs l'interdisciplinarité en acte : enjeux, obstacles, résultats. Ramonville SaintAgne, Érès.

Quidu, M. (2012). Les sciences du sport en mouvement. Innovation et tradition théorique en STAPS. Paris, L'Harmattan.

Stehr N., Weingart P. (2000). Practising Interdisciplinarity. Toronto, University of Toronto Press.

Szpirglas, M. (2006). Gestion des risques et quiproquos, l'apport d'une théorie de la conception. Revue Française de Gestion, 32 (161), 67-90.

TerRaL, P. (2003). La construction sociale des savoirs du monde sportif: sociologie des conceptions épistémiques. Thèse de doctorat non publiée, Université Paris IV-Sorbonne.

Terral, P., Collinet, C. (2007). L'utilisation des savoirs scientifiques par les enseignants d'EPS : entre description, prescription, justification et méta-cognition. Terrain et travaux, 12, 91-106.

Terret, T. (2003). L'institutionnalisation de "l'Aquatic Fitness » aux États-Unis (1984-1992). STAPS, 61, 53-72.

Thibault, J. (1981). Recherche historique et activités physiques et sportives. STAPS, 3, 33-38.

Vinck, D. (2000). Pratiques de l'interdisciplinarité. Mutation des sciences, de l'industrie et de l'enseignement. Grenoble, Presses universitaires de Grenoble.

Wei Chang, C. (2006). Pratiques langagières et stratégies d'action: vers une approche sémio-constructiviste du basket-ball à Taiwan. STAPS, 72, 63-77. 